\title{
Validation of coding algorithms for the identification of patients hospitalized for alcoholic hepatitis using administrative data
}

\author{
Jack XQ Pang ${ }^{1,2}$, Erin Ross ${ }^{1}$, Meredith A. Borman'1, Scott Zimmer ${ }^{3}$, Gilaad G. Kaplan 1,2, Steven J. Heitman 1,2, \\ Mark G. Swain ${ }^{1}$, Kelly W. Burak ${ }^{1,2}$, Hude Quan ${ }^{2}$ and Robert P. Myers ${ }^{1,2^{*}}$
}

\begin{abstract}
Background: Epidemiologic studies of alcoholic hepatitis (AH) have been hindered by the lack of a validated International Classification of Disease (ICD) coding algorithm for use with administrative data. Our objective was to validate coding algorithms for AH using a hospitalization database.

Methods: The Hospital Discharge Abstract Database (DAD) was used to identify consecutive adults ( $\geq 18$ years) hospitalized in the Calgary region with a diagnosis code for AH (ICD-10, K70.1) between 01/2008 and 08/2012. Medical records were reviewed to confirm the diagnosis of AH, defined as a history of heavy alcohol consumption, elevated AST and/or ALT ( $<300 \mathrm{U} / \mathrm{L}$ ), serum bilirubin $>34 \mu \mathrm{mol} / \mathrm{L}$, and elevated INR. Subgroup analyses were performed according to the diagnosis field in which the code was recorded (primary vs. secondary) and AH severity. Algorithms that incorporated ICD-10 codes for cirrhosis and its complications were also examined.

Results: Of 228 potential AH cases, 122 patients had confirmed AH, corresponding to a positive predictive value (PPV) of $54 \%$ (95\% Cl 47-60\%). PPV improved when AH was the primary versus a secondary diagnosis (67\% vs. $21 \%$; $P<0.001$ ). Algorithms that included diagnosis codes for ascites (PPV $75 \%$; $95 \%$ Cl 63-86 \%), cirrhosis (PPV $60 \% ; 47-73 \%$ ), and gastrointestinal hemorrhage (PPV $62 \% ; 51-73 \%$ ) had improved performance, however, the prevalence of these diagnoses in confirmed AH cases was low (29-39\%).
\end{abstract}

Conclusions: In conclusion the low PPV of the diagnosis code for AH suggests that caution is necessary if this hospitalization database is used in large-scale epidemiologic studies of this condition.

\section{Background}

Alcoholic hepatitis (AH) is a clinical syndrome characterized by hepatic dysfunction in the setting of heavy alcohol intake. Rapid onset of jaundice is a cardinal manifestation of $\mathrm{AH}$; other common signs include fever, ascites, muscle wasting and hepatic encephalopathy [1]. $\mathrm{AH}$ is often complicated by infection and hepatorenal syndrome, both of which significantly increase mortality [2-4]. Untreated patients with severe AH, typically defined by a Maddrey discriminant function (DF) $\geq 32$ and/or the presence of hepatic encephalopathy, have a

\footnotetext{
* Correspondence: rpmyers@ucalgary.ca

'Liver Unit, Division of Gastroenterology and Hepatology, University of Calgary, Calgary, AB, Canada

${ }^{2}$ Department of Community Health Sciences, University of Calgary, Calgary, $A B$, Canada

Full list of author information is available at the end of the article
}

particularly poor prognosis with one-month mortality rates ranging from $30 \%$ to $50 \%[5,6]$. In patients with a mild presentation of $\mathrm{AH}$, the risk of progression to cirrhosis is $50 \%$; this risk is highest in patients who continue to abuse alcohol [7].

Population-based studies describing the epidemiology and outcomes of $\mathrm{AH}$ are limited. A Danish study by Sandahl et al. [8] was the first population-based epidemiologic study of $\mathrm{AH}$ and demonstrated an increase in annual incidence among both Danish men and women between 1999 and 2008. Another study described the clinical characteristics and mortality of patients hospitalized in the United States for AH using the Nationwide Inpatient Sample (NIS) database [9]. Both of these studies relied on International Classification of Diseases (ICD) diagnosis codes for $\mathrm{AH}$ in an administrative database for case identification. Although such codes have been validated for other 
non-hepatic and hepatic conditions (e.g. cirrhosis, viral hepatitis, autoimmune liver disease, drug hepatotoxicity) [10-21], their validity for $\mathrm{AH}$ has yet to be confirmed. While the burden of alcohol-related liver disease is increasing in many regions, large-scale studies describing the epidemiology of $\mathrm{AH}$ in Canada have been hindered by the lack of a validated coding algorithm. Therefore, the primary objective of our study was to validate coding algorithms for the identification of patients hospitalized for $\mathrm{AH}$ using administrative data. Our study findings will inform researchers if administrative data can be used for epidemiological studies and surveillance of this condition.

\section{Methods}

\section{Study population}

In this retrospective study, the hospital Discharge Abstract Database (DAD) was queried to identify adults (age $\geq 18$ years) hospitalized in the Calgary region in the province of Alberta, Canada with a primary or secondary diagnosis of AH (ICD-10 diagnosis code, K70.1 [22]) between January 2008 and August 2012. Only the first hospitalization was considered among patients with multiple admissions for AH to avoid selection bias. Specifically, patients diagnosed with $\mathrm{AH}$ are more likely to be diagnosed with $\mathrm{AH}$ again during future admissions, thereby falsely elevating coding validity. The Calgary region included three adult, acute care hospitals and served a catchment population of approximately 1.5 million individuals. Over $99 \%$ of Alberta residents are registrants of the Alberta Health Care Insurance Plan, a universal plan that covers all hospitalization costs [23]. The DAD contains up to 25 diagnoses, 20 procedures, and mortality information on all discharges from Calgary hospitals. This database has been used to examine the epidemiology [24, 25], outcomes [26-33], and coding accuracy $[18,24]$ of various medical conditions. This study protocol and a waiver of consent was approved by the Conjoint Health Research Ethics Board at the University of Calgary.

\section{Data extraction and definition of $\mathrm{AH}$}

Admissions were randomly assigned to one of three physicians experienced in the care of patients with $\mathrm{AH}(\mathrm{MB}$, $E R, J P)$ who reviewed the paper and electronic medical records for each individual to confirm the diagnosis of $\mathrm{AH}$ during their index admission. Using a structured data collection instrument, the following details were recorded: age, sex, year and site of hospitalization, liver biochemistry (serum alanine aminotransferase [ALT], aspartate aminotransferase [AST], gamma-glutamyltranspeptidase [GGT], bilirubin, albumin concentrations, and international normalized ratio [INR]) and serum creatinine at admission, clinical evidence of hepatic encephalopathy (based on the impression of the attending physician) or ascites (detected clinically or radiologically), and self-reported recent average daily alcohol intake. Since alcohol intake varied throughout some medical records, a hierarchical approach was taken. Specifically, data was first taken from consultation notes by addictions specialists where available, followed by the admission history, and otherwise, from patient progress notes. The severity of hepatic dysfunction was described using the Model for End-Stage Liver Disease (MELD) score [34] and Maddrey DF, validated prognostic scoring systems for patients with AH. Patients with a Maddrey DF $\geq 32$ and/or the presence of hepatic encephalopathy were classified as severe [6]. Length of stay (LOS) and mortality (in-hospital and at 90 and 180 days) were also recorded.

Although the reference standard for the diagnosis of $\mathrm{AH}$ includes liver biopsy, this procedure is not part of the routine clinical management of patients with suspected AH in Calgary hospitals. Therefore, patients were considered to have a confirmed diagnosis of $\mathrm{AH}$ if they fulfilled all the following criteria based on history and laboratory investigations at hospital admission: 1) heavy alcohol consumption ( $>196 \mathrm{~g} /$ week or $>56 \mathrm{~g}$ in any day among males, and $>98 \mathrm{~g} /$ week or $>42 \mathrm{~g}$ in any day among females) [35]; 2) elevated serum AST and/or ALT concentration, but $<300 \mathrm{IU} / \mathrm{L}$ (to exclude other disorders associated with acute hepatic dysfunction including acetaminophen toxicity); 3) serum bilirubin $>34 \mu \mathrm{mol} / \mathrm{L}$; 4) elevated INR; and 5) exclusion of other causes of acute hepatic dysfunction (e.g. drug hepatotoxicity, autoimmune hepatitis, ischemic hepatitis, etc.). Patient with missing information on any of the above criteria are considered "Not AH". To confirm the inter-rater agreement of a diagnosis of $\mathrm{AH}$ according to these criteria, 21 patients were randomly selected and all three physicians reviewed their medical records. In cases of disagreement, a consensus was reached.

\section{Statistical analyses}

Between groups comparisons were made using Fisher's exact and $\chi^{2}$ tests for categorical variables, and Wilcoxon rank-sum test for continuous variables. Using data from medical records as the reference standard for the diagnosis of $\mathrm{AH}$, we calculated the positive predictive value (PPV) for each administrative data coding algorithm with exact binomial $95 \%$ confidence intervals (CI). Due to the lack of an unaffected control group, we could not calculate the sensitivity, specificity, or negative predictive values of these algorithms. Subgroup analyses of algorithm accuracy were performed according to the diagnosis field in which the code for $\mathrm{AH}$ was recorded (primary vs. secondary) and AH severity (mild vs. severe), with a severe presentation defined by a Maddrey DF of $\geq 32$ and/or the presence of hepatic encephalopathy. Further stratification 
by fiscal year and admitting hospital was performed to assess for any temporal changes in coding accuracy or heterogeneity between hospitals, which employ different health records coders. In addition, the diagnostic accuracy of algorithms that incorporated ICD-10 diagnosis codes for cirrhosis and its complications (i.e. ascites, gastrointestinal hemorrhage, hepatic encephalopathy, malnutrition, hepatorenal syndrome/renal failure) and alcoholrelated disorders (i.e. alcohol abuse, alcohol dependence, alcohol withdrawal, and pancreatitis) were examined (see Appendix for codes). Algorithms that accounted for the number of associated conditions were also assessed. Finally, inter-rater agreement for the diagnosis of $\mathrm{AH}$, with subgroup analyses according to disease severity, was calculated using the kappa statistic [36]. All analyses were performed using Stata version 11.0 (StataCorp; College Station, TX, USA). Two-sided $P$-values less than 0.05 were considered statistically significant.

\section{Results}

\section{Patient characteristics}

A total of 228 patients were hospitalized in the Calgary region between January 2008 and August 2012 with a diagnosis code for $\mathrm{AH}(71 \%[n=161]$ as the primary diagnosis). Their characteristics are outlined in Table 1. The median age was 49 years (interquartile range [IQR] 43-55) and $61 \%$ of the cohort was male. A similar proportion was hospitalized at each of the three adult hospitals in Calgary $(P=0.69)$. The majority of cases $(62 \%, n=142)$ had a severe presentation. Overall, median length of stay was 7 days (IQR 5-15) and in-hospital mortality was $6 \%(n=13)$. Compared with those with a mild presentation, patients with a severe presentation had a prolonged median LOS (5 vs. 10 days; $P<0.001$ ) and greater in-hospital mortality ( $0 \%$ vs. $9 \% ; P=0.002)$.

Validation of an $\mathrm{AH}$ diagnosis based on administrative data Of the 228 potential AH cases, 122 patients had confirmed $\mathrm{AH}$ based on medical record review, corresponding to a PPV of $54 \%$ (95\% CI 47-60\%). Overall, inter-rater agreement for an $\mathrm{AH}$ diagnosis was good (kappa $0.86 ; P<0.001$ ), and higher among patients with a severe (kappa $0.88 ; P<0.001$ ) versus mild (kappa 0.80; $P<0.002)$ presentation. Compared to unconfirmed cases, patients with confirmed $\mathrm{AH}$ had greater liver disease severity according to the Maddrey DF (45 vs. $12 ; P<0.001$ ) and MELD scores (21 vs. $13 ; P<0.001$ ); a higher prevalence of ascites (38 \% vs. $14 \% ; P<0.001$ ); a lower prevalence of alcohol withdrawal (16\% vs. $26 \%$; $P=0.05)$; and greater 90-day and 180-day mortality (Table 1). Patients with confirmed $\mathrm{AH}$ also had greater median daily alcohol intake (121 vs. 112 g; $P=0.024$ ), however, there was no significant difference in the proportion of patients that had daily alcohol intake within the top quartile (24\% vs. $25 \%$;
$P=0.87$ ). Differences between confirmed and unconfirmed $\mathrm{AH}$ cases were not observed with respect to age, sex, admitting hospital, year of admission, or the remaining cirrhosis and alcohol-related complications (Table 1).

\section{Subgroup analyses of $\mathrm{AH}$ coding algorithm validity}

Among confirmed AH cases, 89 \% (108/122) had an AH code recorded as the primary diagnosis compared with only $50 \%(53 / 106)$ among unconfirmed cases $(P<0.001)$. The PPV improved from $54 \%$ overall to $67 \%$ (95 \% CI 59$74 \%$ ) when $\mathrm{AH}$ was the primary diagnosis versus $21 \%$ (95\% CI 12-33 \%) when AH was recorded as a secondary diagnosis $(P<0.001)$. A severe presentation was also more common in patients with confirmed $\mathrm{AH}$ versus unconfirmed cases (80\% [97/122] vs. $43 \%$ [45/106]; $P<0.001)$. Accordingly, the PPV of an $\mathrm{AH}$ diagnosis was higher among severe compared to mild cases (68\% [95 \% CI 60$76 \%$ ] vs. $29 \%$ [95 \% CI 20-40\%]; $P<0.001)$. When stratified by year of hospitalization, the PPV was highest in 2012 (70 \%; 95 \% CI 54-83 \%) compared to 2008 through 2011 (45\% to $54 \%$ ), however, this difference was not statistically significant $(P=0.17)$. Comparison of the PPV between admitting hospitals, which ranged from $50 \%$ to $57 \%$, showed no significant differences between centres $(P=0.69)$.

The PPVs of diagnostic algorithms that included codes for cirrhosis and alcohol-related conditions in the administrative data are shown in Table 2. The PPVs tended to be higher for algorithms that included ascites (75\%; $95 \%$ CI 63-86 \%), gastrointestinal hemorrhage (62\%; $95 \%$ CI 51-73\%), and cirrhosis (60\%; $95 \%$ CI $47-$ $73 \%)$. In general, these PPVs improved when restricted to cases with an $\mathrm{AH}$ code in the primary diagnosis field and those with severe presentations (Table 2). However, the prevalence of these conditions in patients with confirmed $\mathrm{AH}$ was low, ranging from $29 \%$ for cirrhosis to $39 \%$ for gastrointestinal hemorrhage. The prevalence of codes for other cirrhosis and alcohol-related disorders were generally too low or their inclusion did not enhance the PPV of the algorithms (Table 2). For example, a diagnosis code for hepatic encephalopathy was recorded in only $2.5 \%$ of confirmed AH cases ( $4.2 \%$ of severe cases), and thus, the PPV of an algorithm including both an $\mathrm{AH}$ and hepatic encephalopathy code was only $50 \%$ (95 \% CI 12-88 \%). Stratification by year and hospital of admission did not indicate any consistent differences between PPVs for these algorithms (data not shown).

Table 3 outlines the performance characteristics of algorithms according to the number of cirrhosis and alcohol-related conditions recorded in the administrative data in addition to an $\mathrm{AH}$ diagnosis code. In patients with three or more associated conditions, the PPV for confirmed $\mathrm{AH}$ improved to $62 \%$ to $79 \%$; however, only $6 \%$ to $34 \%$ of confirmed cases had this number of additional diagnoses in their administrative data. 
Table 1 Characteristics of the study population

\begin{tabular}{|c|c|c|c|c|}
\hline & Entire cohort $(n=228)$ & Confirmed AH cases $(n=122)$ & Unconfirmed AH cases $(n=106)$ & $P$-value \\
\hline Age, years & $49(43-55)$ & $49(42-55)$ & $49(44-58)$ & 0.33 \\
\hline Male & $61 \%(138)$ & $60 \%(73)$ & $61 \%(65)$ & 0.89 \\
\hline Self-reported alcohol consumption, g/day & $119(81-224)$ & $121(84-233)$ & $112(56-208)$ & 0.024 \\
\hline Excessive intake $^{a}$ & $91 \%(208)$ & $100 \%(122)$ & $81 \%(86)$ & $<0.001$ \\
\hline Proportion in the highest intake quartile & $25 \%(50)$ & $25 \%(29)$ & $24 \%(21)$ & 0.87 \\
\hline Admission hospital & & & & 0.69 \\
\hline 1 & $39 \%(90)$ & $42 \%(51)$ & $37 \%(39)$ & \\
\hline 2 & $30 \%(68)$ & $29 \%(36)$ & $31 \%(32)$ & \\
\hline 3 & $31 \%(70)$ & $29 \%(35)$ & $33 \%(35)$ & \\
\hline Year of admission & & & & 0.16 \\
\hline 2008 & $25 \%(56)$ & $22 \%(27)$ & $27 \%(29)$ & \\
\hline 2009 & $17 \%(40)$ & $15 \%(18)$ & $21 \%(22)$ & \\
\hline 2010 & $18 \%(41)$ & $17 \%(21)$ & $19 \%(20)$ & \\
\hline 2011 & $21 \%(48)$ & $21 \%(26)$ & $21 \%(22)$ & \\
\hline 2012 & $19 \%(43)$ & $25 \%(30)$ & $12 \%(13)$ & \\
\hline \multicolumn{5}{|l|}{ Biochemical status } \\
\hline Maddrey DF & $29(13-52)$ & $45(26-62)$ & $12(5-30)$ & $<0.001$ \\
\hline MELD & $18(13-23)$ & $21(18-24)$ & $13(8-19)$ & $<0.001$ \\
\hline Severe $^{\mathrm{b}}$ & $62 \%(142)$ & $80 \%(97)$ & $42 \%(45)$ & $<0.001$ \\
\hline \multicolumn{5}{|l|}{ Associated conditions } \\
\hline Cirrhosis & $25 \%(58)$ & $29 \%(35)$ & $22 \%(23)$ & 0.29 \\
\hline Ascites & $27 \%(61)$ & $38 \%(46)$ & $14 \%(15)$ & $<0.001$ \\
\hline Gl hemorrhage & $34 \%(77)$ & $39 \%(48)$ & $27 \%(29)$ & 0.07 \\
\hline Hepatic encephalopathy & $2.6 \%(6)$ & $2.5 \%(3)$ & $2.8 \%(3)$ & 1 \\
\hline Malnutrition & $3.0 \%(7)$ & $4.1 \%(5)$ & $1.9 \%(2)$ & 0.45 \\
\hline HRS/renal failure & $16 \%(36)$ & $18 \%(22)$ & $13 \%(14)$ & 0.37 \\
\hline Pancreatitis & $7.9 \%(18)$ & $4.9 \%(6)$ & $11 \%(12)$ & 0.09 \\
\hline Alcohol abuse & $36 \%(81)$ & $38 \%(46)$ & $33 \%(35)$ & 0.49 \\
\hline Alcohol dependence & $26 \%(60)$ & $28 \%(34)$ & $25 \%(26)$ & 0.65 \\
\hline Alcohol withdrawal & $21 \%(47)$ & $16 \%(19)$ & $26 \%(28)$ & 0.05 \\
\hline \multicolumn{5}{|l|}{ Mortality } \\
\hline In-hospital & $5.7 \%(13)$ & $7.4 \%$ (9) & 3.7 \% (4) & 0.27 \\
\hline 90 days & $13 \%(29)$ & $17 \%(21)$ & 8.5 \% (9) & 0.045 \\
\hline 180 day & $17 \%(38)$ & $23 \%(28)$ & $11 \%(12)$ & 0.007 \\
\hline
\end{tabular}

Data are presented as median (IQR) or \% (n). DF discriminant function, Gl gastrointestinal, HRS hepatorenal syndrome; MELD, Model for End-Stage Liver Disease a As defined in the Methods

${ }^{b}$ Severe presentation is characterized by a Maddrey DF of $\geq 32$ and/or the presence of hepatic encephalopathy

\section{Discussion}

In this population-based study, we assessed the validity of ICD diagnosis coding algorithms for $\mathrm{AH}$ in a Canadian hospitalization database. Our finding of a low PPV for AH (54 \%) suggests that AH was not accurately and completely coded in the administrative data and caution must be exercised if the DAD is used for largescale epidemiologic studies of AH. Under the assumption that the PPV remains relatively constant over time (as observed in our study), the DAD could be used to assess temporal trends in $\mathrm{AH}$ admissions. However, the exact incidence for any given year based on this data is likely to be erroneous. Administrative data could also be used as a screening tool for potential cases with $\mathrm{AH}$. In this situation, confirmation of the presence of $\mathrm{AH}$ requires additional clinical information such as that obtainable via a review of medical records. In light of our findings, the validity of previous studies that used 
Table 2 Prevalence and performance characteristics of algorithms including an alcoholic hepatitis code and codes for associated conditions

\begin{tabular}{|c|c|c|c|c|c|c|c|}
\hline \multirow[b]{2}{*}{ Condition } & \multicolumn{2}{|c|}{ Prevalence \% ( $n)$} & \multicolumn{5}{|c|}{ Positive predictive value (PPV) \% $(95 \% \mathrm{Cl})$} \\
\hline & $\begin{array}{l}\text { Overall } \\
(n=22\end{array}$ & $\begin{array}{l}\text { Confirmed } \mathrm{AH} \\
(n=122)\end{array}$ & $\begin{array}{l}\text { Overall } \\
(n=228\end{array}$ & $\begin{array}{l}\text { AH as Primary } \\
\text { Diagnosis }(n=161)\end{array}$ & $\begin{array}{l}\text { AH as Secondary } \\
\text { Diagnosis }(n=67)\end{array}$ & $\begin{array}{l}\text { Severe AH } \\
(n=142)\end{array}$ & $\begin{array}{l}\text { Mild AH } \\
(n=86)\end{array}$ \\
\hline Ascites & $27 \%(61)$ & $38 \%(46)$ & $75 \%(63-86)$ & $78 \%(65-89)$ & $6 \%(26-88)$ & $83 \%(70-93)$ & $46 \%(19-75)$ \\
\hline Gl hemmorrhage & $34 \%(77)$ & $39 \%(48)$ & $62 \%(51-73)$ & $76 \%(62-87)$ & $30 \%(13-53)$ & $80 \%(66-90)$ & $30 \%(14-50)$ \\
\hline Hepatic encephalopathy & $2.6 \%(6)$ & $2.5 \%(3)$ & $50 \%(12-88)$ & $100 \%(16-100)$ & $25 \%(1-81)$ & $50 \%(12-88)$ & - \\
\hline Cirrhosis & $25 \%(58)$ & $29 \%(35)$ & $60 \%(47-73)$ & $70 \%(53-83)$ & $39 \%(17-64)$ & $69 \%(53-82)$ & $31 \%(9-61)$ \\
\hline Alcoholic hepatic failure & $4.8 \%(11)$ & $8.2 \%(10)$ & $91 \%(59-100)$ & $89 \%(52-100)$ & $44 \%(20-70)$ & $90 \%(55-100)$ & $33 \%(10-65)$ \\
\hline Malnutrition & $3.0 \%(7)$ & $4.1 \%(5)$ & $71 \%(29-96)$ & $60 \%(15-95)$ & $100 \%(16-100)$ & $80 \%$ (28-99) & $50 \%(1-99)$ \\
\hline HRS/ Renal failure & $16 \%(36)$ & $18 \%(22)$ & $61 \%(43-77)$ & $67 \%(46-83)$ & $44 \%(14-79)$ & $68 \%(48-84)$ & $38 \%(9-76)$ \\
\hline Pancreatitis & $7.9 \%(18)$ & $4.9 \%(6)$ & $33 \%(13-59)$ & $63 \%(24-91)$ & $10 \%(0-45)$ & $86 \%(42-100)$ & $0 \%(0-28)$ \\
\hline Alcohol abuse & $35 \%(81)$ & 38 \% (46) & $57 \%(43-68)$ & $68 \%(54-79)$ & $27 \%(11-50)$ & $70 \%(56-82)$ & $32 \%(16-52)$ \\
\hline Alcohol dependence & $26 \%(60)$ & $28 \%(34)$ & $57 \%(43-69)$ & $74 \%(60-86)$ & $17 \%(4-41)$ & 76 \% (59-88) & $26 \%(10-48)$ \\
\hline Alcohol withdrawal & $21 \%(47)$ & $16 \%(19)$ & $40 \%(26-56)$ & $62 \%(41-80)$ & $14 \%(3-36)$ & $62 \%(41-80)$ & $14 \%(3-36)$ \\
\hline
\end{tabular}

GI gastrointestinal, HRS hepatorenal syndrome

administrative data to study the epidemiology and outcomes of AH should be scrutinized. In one study, Sandahl and colleagues used this ICD-10 code to identify AH cases from the Danish National Registry of Patients and describe its incidence and associated mortality [8]. In total, 1,951 suspected cases of AH were identified between 1999 and 2008. The annual incidence increased from 37 to 46 per million population in men and from 24 to 34 per million in women. However, according to our results, close to half of the patients identified in this study may not have been true cases of AH. This study also reported 84-day mortality ranging from $14 \%$ to $24 \%$, similar to the 90 -day mortality rate of $17 \%$ for confirmed $\mathrm{AH}$ in our study. In another study, Liangpunsakul used the Nationwide Inpatient Sample hospitalization database to report the outcomes of patients hospitalized for $\mathrm{AH}$ in the United States with case identification using the ICD-9 code for this condition (571.1) [9]. The study showed that AH represented $0.71 \%$ of all hospital admissions in 2007 and

Table 3 Performance characteristics for algorithms for alcoholic hepatitis according to the number of associated conditions ${ }^{\text {a }}$

\begin{tabular}{llll}
\hline $\begin{array}{l}\text { Number of } \\
\text { conditions }\end{array}$ & ${ }^{a}$ & Prevalence \% $(n)$ & $\begin{array}{l}\text { Positive predictive } \\
\text { value (PPV) } \% \\
(95 \% \text { Cl) }\end{array}$ \\
\hline 0 & $11 \%(24)$ & $7.4 \%(9)$ & $38 \%(19-59)$ \\
$\geq 1$ & $89 \%(204)$ & $93 \%(113)$ & $55 \%(48-62)$ \\
$\geq 2$ & $61 \%(139)$ & $65 \%(79)$ & $57 \%(48-65)$ \\
$\geq 3$ & $30 \%(68)$ & $34 \%(42)$ & $62 \%(49-73)$ \\
$\geq 4$ & $13 \%(29)$ & $19 \%(23)$ & $79 \%(60-92)$ \\
$\geq 5$ & $4 \%(9)$ & $5.7 \%(7)$ & $78 \%(40-97)$ \\
\hline
\end{tabular}

${ }^{a}$ Associated conditions as shown in Table 2. Prevalent cases represent those with an $\mathrm{AH}$ diagnosis code plus codes for the associated conditions reported an in-hospital mortality rate of $6.8 \%$. Although our study validated only the ICD-10 coding classification (as ICD-9 codes were unavailable), there is no clear rationale for the accuracy to differ among the two classification systems both of which include a single code for AH. Moreover, a study by Quan and colleagues that used the DAD (as in our study) demonstrated that the coding accuracies between the ICD-9 and ICD-10 classifications for liver diseases are very similar (AH was not examined specifically in this study) [37]. Therefore, our results should be generalizable to databases using either the ICD-9 or ICD-10 classifications.

The diagnosis field in which the code for $\mathrm{AH}$ was recorded in the administrative database (i.e. primary vs. secondary) had a significant impact on algorithm validity. Indeed, the majority ( $89 \%$ ) of confirmed $\mathrm{AH}$ cases had the AH code recorded as the primary diagnosis (vs. $50 \%$ among non-AH hospitalizations). Accordingly, the PPV improved from $54 \%$ to $67 \%$ when the cohort was restricted to those with $\mathrm{AH}$ as the primary diagnosis compared with one of the secondary diagnosis fields. Patients with a severe presentation were also more likely to have AH recorded as the primary diagnosis. Specifically, $77 \%$ of cases with hepatic encephalopathy and/or a Maddrey DF $\geq 32$ had AH as a primary diagnosis compared with only $59 \%$ of those with mild hepatic dysfunction. Algorithms that also included diagnosis codes for ascites or gastrointestinal hemorrhage had improved performance, particularly when restricted to cases with a primary diagnosis of $\mathrm{AH}$ in which PPVs of $76 \%$ to $78 \%$ were observed. The corollary is that studies focused on patients with severe $\mathrm{AH}$ may potentially identify these cases from administrative databases by restricting to cases with $\mathrm{AH}$ as the primary diagnosis plus these 
associated conditions. However, the prevalence of these cirrhosis-related complications was low enough $(<40 \%)$ in confirmed $\mathrm{AH}$ cases that any study employing this methodology will have reduced sensitivity for the identification of all relevant cases. Based on the sub-optimal accuracy observed in our study, we would advise that any $\mathrm{AH}$ case identified in this manner be confirmed via a review of medical records.

In addition to examining the impacts of coding details and disease severity on the validity of an $\mathrm{AH}$ code in the administrative data, we studied the effects of year and hospital of admission. Despite the presence of different health records coders at the three hospitals in our region, coding validity was similar supporting the generalizability of our findings. Moreover, the PPV of an AH code did not differ across study years, potentially supporting the temporal trends in disease incidence suggested by the study of Sandahl and colleagues [8]. In this regard, we did not observe an increase in the number of admissions over time although the sample size of our study was limited.

While previous studies have confirmed the validity of administrative data for the identification of patients with various liver disorders [19, 38, 39], our results suggest that caution is needed when using these data sources to study $\mathrm{AH}$. However, the major challenge with our analysis relates to the diagnostic definition for AH. Specifically, $\mathrm{AH}$ represents a spectrum ranging from mild abnormalities in liver biochemistry to life-threatening liver failure due to abusive alcohol consumption when other causes of liver disease (e.g. viral hepatitis and drug hepatotoxicity) have been excluded. Although $\mathrm{AH}$ has characteristic histological findings including steatosis, ballooned hepatocytes, Mallory bodies, lobular neutrophilc inflammation, and centrizonal fibrosis [40], liver biopsy is not routinely performed in our region, nor are these findings specific (e.g. they may be seen in patients with non-alcoholic steatohepatitis). The diagnostic criteria suggested by Lucey et al. (i.e. elevated AST but $<300 \mathrm{IU} / \mathrm{L}$, AST to ALT ratio $>2$, serum bilirubin $>86 \mu \mathrm{mol} / \mathrm{L}$, elevated INR, and neutrophilia in patients with ascites and a history of heavy alcohol use) [1] are very stringent and would mostly capture only patients with severe $\mathrm{AH}$. In light of this fact, we utilized a less rigorous definition of $\mathrm{AH}$ that allowed us to identify a wider spectrum of suspected $\mathrm{AH}$ cases. Moreover, since AST is not part of standard liver biochemical profiles in our region and due to the high correlation between AST and ALT, we considered ALT in place of AST when necessary. Nevertheless, since such a biochemically focused, even more lenient, case definition is generally not strictly followed in clinical practice, patients who do not meet all of these criteria may still be diagnosed with $\mathrm{AH}$ by their physician thereby contributing to the sub-optimal accuracy of the diagnosis code observed in our study.
Among the 106 patients who did not fulfill our diagnostic criteria for confirmed AH, 31 patients clearly did not have AH upon medical records review. The diagnosis for these patients includes cirrhosis, sepsis, recent history of $\mathrm{AH}$, pancreatitis, acetaminophen toxicity, cocaine use, heart failure and alcohol intoxication.

Taking into consideration that our definition of $\mathrm{AH}$ may not be strictly followed by physicians, we performed a sensitivity analysis using a loosened definition of $\mathrm{AH}$ that confirmed diagnosis of $\mathrm{AH}$ if patients fulfilled both of the following criteria: heavy alcohol consumption ( $>196 \mathrm{~g} /$ week or $>56 \mathrm{~g}$ in any day among males, and $>98 \mathrm{~g} /$ week or $>42 \mathrm{~g}$ in any day among females) and exclusion of other causes of acute hepatic dysfunction (e.g. drug hepatotoxicity, autoimmune hepatitis, ischemic hepatitis, etc.). With this loosened diagnostic criteria, PPV improved to 73 \% (95 \% CI 67-79\%), up from $54 \%$. This improved PPV remains sub-optimal, thus further supporting our initial findings that $\mathrm{AH}$ was not accurately and completely coded in the administrative data. Realizing the limitations of both sets of diagnostic criteria, one could infer that the true PPV for the AH diagnosis code lies within $54 \%$ to $73 \%$.

Our study has several limitations that warrant discussion. First, we lack data for a control group representative of the general hospitalized population. Without controls, the sensitivity, specificity and NPVs of the diagnosis code for $\mathrm{AH}$ (and related algorithms) cannot be calculated. Second, since this is a retrospective study, the reliability of patients' self-reported alcohol intake is questionable. On numerous occasions, multiple descriptions of alcohol consumption were recorded in the medical record. Anecdotally, patients tended to admit to greater alcohol intake when questioned by addictions specialists. As a result, we utilized a hierarchical approach to record alcohol consumption (see Methods). Since the amount of alcohol intake is a vital criterion in the reference standard for an $\mathrm{AH}$ diagnosis, underreporting may have led to an underestimation of $\mathrm{AH}$ in our study.

\section{Conclusions}

In conclusion, the validity of ICD-10 coding for AH in this Canadian hospitalization database appears sub-optimal. Although the accuracy of coding algorithms improved when restricted to patients with $\mathrm{AH}$ in the primary diagnosis field or with codes indicative of hepatic complications (e.g. ascites and gastrointestinal haemorrhage), the use of administrative data in epidemiologic studies of $\mathrm{AH}$ should be undertaken cautiously. If administrative data is used for case identification, confirmation of the diagnosis via medical record review is recommended.

\section{Availability of data and materials}

Not applicable. 


\section{Appendix}

Table 4 Codes used to identify associated conditions

\begin{tabular}{lll}
\hline Condition & ICD-10 diagnosis codes & CCl procedure codes [41] \\
\hline Alcoholic hepatitis & K70.1 & 1OT52HA, 1OT52HAT, 1OT52LAT \\
Ascites & R18 & 1NA13BAH, 1NA13BAX, 1NA13BAF, 1NF13BAK \\
Gl hemorrhage & K92.0, K92.1, K92.2, K62.5, 185.0, 185.9, 198.20, 198.21, 198.3, & \\
& K25.3, K25.4, K25.7, K25.9, K26.4, K26.9, K27.9, K22.6, K22.1 & \\
Hepatic encephalopathy & K72 \\
Cirrhosis & K70.3, K74.6 \\
Alcoholic hepatic failure & K70.4 \\
Malnutrition & E41, E43, E46 \\
HRS/renal failure & K76.7, N17.0, N17.8, N17.9 & 1PZ21HPD, 1PZ21HQB \\
Pancreatitis & K85, K85.2, K85.9, K86.0, K86.1 \\
Alcohol abuse & F10.1 \\
Alcohol dependence & F10.2 \\
Alcohol withdrawal & F10.3
\end{tabular}

Gl gastrointestinal, HRS hepatorenal syndrome, CCI Canadian Classification of Health Interventions

\section{Abbreviations}

AH: Alcoholic hepatitis; ALT: Alanine aminotransferase; AST: Aspartate aminotransferase; Cl: Confidence interval; $\mathrm{ClHI}$ : Canadian Institute of Health Information; Gl: Gastrointestinal; HE: Hepatic encephalopathy; ICD: International statistical classification of disease; INR: International normalized ratio; IQR: Interquartile range; NIS: Nationwide Inpatient Sample; PPV: Positive predictive value.

\section{Competing interests}

Dr. Myers was supported by a salary support award from the Canadian Institutes for Health Research (CIHR). Dr. Kaplan is supported by salary support awards from CIHR and Alberta Innovates-Health Solutions (AIHS). Dr. Swain is supported by the Cal Wenzel Family Foundation Chair in Hepatology. Dr. Quan is supported by a salary support award from AlHS. Dr. Borman is supported by a Canadian Association for the Study of the Liver/Nertex Clinical Hepatology Fellowship. Dr. Heitman was supported by an award from the Noel Hershfield Professorship in Therapeutic Endoscopy.

\section{Authors' contributions}

JP was involved in the design of the study, data extraction, data analysis, and writing of the manuscript. RM, ER, MB and MS were involved in the conception and design of the study. ER, MB, SZ assisted in data collection for the study. GK, SH, HQ and KB provided assistance for the analysis of this study. All authors participated in the final development of the manuscript and approved of its contents.

\section{Authors' information}

Not applicable.

\section{Acknowledgment}

This study is based in part on data provided by Alberta Health. The interpretation and conclusions contained herein are those of the researchers and do not necessarily represent the views of the Government of Alberta. Neither the Government nor Alberta Health express any opinion in relation to this study.

\section{Funding}

This study was funded in part by grants from Alberta Innovates-Health Solution, Canadian Institutes of Health Research, and the Canadian Liver Foundation.

\section{Author details}

${ }^{1}$ Liver Unit, Division of Gastroenterology and Hepatology, University of Calgary, Calgary, AB, Canada. ${ }^{2}$ Department of Community Health Sciences, University of Calgary, Calgary, AB, Canada. ${ }^{3}$ Medical Services, Alberta Health Services, Calgary, AB, Canada.

Received: 28 January 2015 Accepted: 9 September 2015 Published online: 11 September 2015

\section{References}

1. Lucey MR, Mathurin P, Morgan TR. Alcoholic hepatitis. N Engl J Med. 2009:360(26):2758-69. http://dx.doi.org/10.1056/NEJMra0805786.

2. Akriviadis E, Botla R, Briggs W, Han S, Reynolds T, Shakil O. Pentoxifylline improves short-term survival in severe acute alcoholic hepatitis: a doubleblind, placebo-controlled trial. Gastroenterology. 2000;119(6):1637-48.

3. Mendenhall CL, Moritz TE, Roselle GA, Morgan TR, Nemchausky BA, Tamburro $\mathrm{CH}$, et al. A study of oral nutritional support with oxandrolone in malnourished patients with alcoholic hepatitis: results of a Department of Veterans Affairs cooperative study. Hepatology. 1993;17(4):564-76.

4. Verma S, Ajudia K, Mendler M, Redeker A. Prevalence of septic events, type 1 hepatorenal syndrome, and mortality in severe alcoholic hepatitis and utility of discriminant function and MELD score in predicting these adverse events. Dig Dis Sci. 2006;51(9):1637-43.

5. Carithers Jr RL, Herlong HF, Diehl AM, Shaw EW, Combes B, Fallon HJ, et al. Methylprednisolone therapy in patients with severe alcoholic hepatitis. A randomized multicenter trial. Ann Intern Med. 1989;110(9):685-90.

6. Maddrey WC, Boitnott JK, Bedine MS, Weber Jr FL, Mezey E, White Jr RI. Corticosteroid therapy of alcoholic hepatitis. Gastroenterology. 1978;75(2):193-9.

7. O'Shea RS, Dasarathy S, McCullough AJ. Practice Guideline Committee of the American Association for the Study of Liver D, Practice Parameters Committee of the American College of G. Alcoholic liver disease. Hepatology. 2010;51(1):307-28. http://dx.doi.org/10.1002/hep.23258.

8. Sandahl TD, Jepsen $P$, Thomsen $K L$, Vilstrup $H$. Incidence and mortality of alcoholic hepatitis in Denmark 1999-2008: a nationwide population based cohort study. J Hepatol. 2011;54(4):760-4. http://dx.doi.org/10.1016/ j.jhep.2010.07.016.

9. Liangpunsakul S. Clinical characteristics and mortality of hospitalized alcoholic hepatitis patients in the United States. J Clin Gastroenterol. 2011;45(8):714-9. http://dx.doi.org/10.1097/MCG.0b013e3181fdef1d.

10. Allen-Dicker J, Klompas M. Comparison of electronic laboratory reports, administrative claims, and electronic health record data for acute viral 
hepatitis surveillance. J Public Health Manag Pract. 2012;18(3):209-14. doi:10.1097/PHH.0b013e31821f2d73.

11. Drahos J, Vanwormer JJ, Greenlee RT, Landgren O, Koshiol J. Accuracy of ICD-9-CM codes in identifying infections of pneumonia and herpes simplex virus in administrative data. Ann Epidemiol. 2013;23(5):291-3. doi:10.1016/ j.annepidem.2013.02.005.

12. Goetz MB, Hoang T, Kan VL, Rimland D, Rodriguez-Barradas M. Development and Validation of an Algorithm to Identify Patients Newly Diagnosed with HIV Infection from Electronic Health Records. AIDS Res Hum Retroviruses. 2014 doi:10.1089/AID.2013.0287.

13. Goldberg D, Lewis J, Halpern S, Weiner M, Lo Re 3rd V. Validation of three coding algorithms to identify patients with end-stage liver disease in an administrative database. Pharmacoepidemiol Drug Saf. 2012;21(7):765-9. doi:10.1002/pds.3290.

14. Goldberg DS, Lewis JD, Halpern SD, Weiner MG, Lo Re 3rd V. Validation of a coding algorithm to identify patients with hepatocellular carcinoma in an administrative database. Pharmacoepidemiol Drug Saf. 2013;22(1):103-7. doi:10.1002/pds.3367.

15. Ma C, Crespin M, Proulx MC, DeSilva S, Hubbard J, Prusinkiewicz M, et al. Postoperative complications following colectomy for ulcerative colitis: a validation study. BMC Gastroenterol. 2012;12:39. do:10.1186/1471-230X-12-39.

16. McCormick N, Lacaille D, Bhole V, Avina-Zubieta JA. Validity of myocardial infarction diagnoses in administrative databases: a systematic review. PLoS One. 2014;9(3), e92286. doi:10.1371/journal.pone.0092286.

17. Molodecky NA, Myers RP, Barkema HW, Quan H, Kaplan GG. Validity of administrative data for the diagnosis of primary sclerosing cholangitis: a population-based study. Liver Int. 2011;31(5):712-20. doi:10.1111/j.14783231.2011.02484.x.

18. Myers RP, Leung Y, Shaheen AA, Li B. Validation of ICD-9-CM/ICD-10 coding algorithms for the identification of patients with acetaminophen overdose and hepatotoxicity using administrative data. BMC Health Serv Res. 2007:7:159. doi:10.1186/1472-6963-7-159.

19. Myers RP, Shaheen AA, Fong A, Wan AF, Swain MG, Hilsden RJ, et al. Validation of coding algorithms for the identification of patients with primary biliary cirrhosis using administrative data. Can J Gastroenterol. 2010;24(3):175-82.

20. Nehra MS, Ma Y, Clark C, Amarasingham R, Rockey DC, Singal AG. Use of administrative claims data for identifying patients with cirrhosis. J Clin Gastroenterol. 2013;47(5):e50-4. doi:10.1097/MCG.0b013e3182688d2f

21. Ronksley PE, Tonelli M, Quan H, Manns BJ, James MT, Clement FM, et al. Validating a case definition for chronic kidney disease using administrative data. Nephrol Dial Transplant. 2012;27(5):1826-31. doi:10.1093/ndt/gfr598.

22. World Health Organization. International Statistical Classification of Diseases and Related Health Problems 10th Revision Instruction manual. 2010 ed. Geneva: World Health Organization.

23. Data Disclosure Handbook. Alberta Health and Wellness; 2003. p. 1-15.

24. Myers RP, Liu M, Shaheen AA. The burden of hepatitis $C$ virus infection is growing: a Canadian population-based study of hospitalizations from 1994 to 2004. Can J Gastroenterol. 2008;22(4):381-7.

25. Kaplan GG, Gregson DB, Laupland KB. Population-based study of the epidemiology of and the risk factors for pyogenic liver abscess. Clin Gastroenterol Hepatol. 2004;2(11):1032-8.

26. Myers RP, Shaheen AA, Li B, Dean S, Quan H. Impact of liver disease, alcohol abuse, and unintentional ingestions on the outcomes of acetaminophen overdose. Clin Gastroenterol Hepatol. 2008:6(8):918-25. doi:10.1016/ j.cgh.2008.02.053.

27. Myers RP, Fong A, Shaheen AA. tilization rates, complications and costs of percutaneous liver biopsy: a population-based study including 4275 biopsies. Liver Int. 2008;28(5):705-12. doi:10.1111/j.1478-3231.2008.01691.x.

28. Myers RP, Shaheen AA, Fong A, Burak KW, Wan A, Swain MG, et al. Epidemiology and natural history of primary biliary cirrhosis in a Canadian health region: a population-based study. Hepatology. 2009;50(6):1884-92. doi:10.1002/hep.23210.

29. Pang JX, Pradhan F, Zimmer S, Niu S, Crotty P, Tracey J, et al. The feasibility and reliability of transient elastography using Fibroscan (R): a practice audit of 2335 examinations. Can J Gastroenterol Hepatol. 2014;28(3):143-9.

30. Pang JX, Zimmer S, Niu S, Crotty P, Tracey J, Pradhan F, et al. Liver stiffness by transient elastography predicts liver-related complications and mortality in patients with chronic liver disease. PLoS One. 2014;9(4), e95776. doi:10.1371/journal.pone.0095776.
31. de Silva S, Ma C, Proulx MC, Crespin M, Kaplan BS, Hubbard J, et al Postoperative complications and mortality following colectomy for ulcerative colitis. Clin Gastroenterol Hepatol. 2011;9(11):972-80. doi:10.1016/j.cgh.2011.07.016.

32. Kaplan GG, Seow CH, Ghosh S, Molodecky N, Rezaie A, Moran GW, et al. Decreasing colectomy rates for ulcerative colitis: a population-based time trend study. Am J Gastroenterol. 2012;107(12):1879-87. doi:10.1038/ajg.2012.333.

33. Soon IS, Wrobel I, de Bruyn JC, Sauve R, Sigalet DL, Kaplan BS, et al. Postoperative complications following colectomy for ulcerative colitis in children. J Pediatr Gastroenterol Nutr. 2012;54(6):763-8. doi:10.1097/ MPG.0b013e318245265c

34. Dunn W, Jamil LH, Brown LS, Wiesner RH, Kim WR, Menon KV, et al. MELD accurately predicts mortality in patients with alcoholic hepatitis. Hepatology. 2005;41(2):353-8. doi:10.1002/hep.20503.

35. Moderate \& Binge Drinking. National Institute on Alcohol Abuse and Alcoholism. http://www.niaaa.nih.gov/alcohol-health/overview-alcoholconsumption/moderate-binge-drinking. Accessed May 2014.

36. Sim J, Wright CC. The kappa statistic in reliability studies: use, interpretation, and sample size requirements. Phys Ther. 2005;85(3):257-68.

37. Quan H, Li B, Saunders LD, Parsons GA, Nilsson Cl, Alibhai A, et al. Assessing validity of ICD-9-CM and ICD-10 administrative data in recording clinical conditions in a unique dually coded database. Health Serv Res. 2008;43(4):1424-41.

38. Karam V, Gunson B, Roggen F, Grande L, Wannoff W, Janssen M, et al. Quality control of the European Liver Transplant Registry: results of audit visits to the contributing centers. Transplantation. 2003;75(12):2167-73. doi:10.1097/01.TP.0000080271.20145.07.

39. Steinke DT, Weston TL, Morris AD, MacDonald TM, Dillon JF. The epidemiology of liver disease in Tayside database: a population-based record-linkage study. J Biomed Inform. 2002;35(3):186-93.

40. Altamirano J, Miquel R, Katoonizadeh A, Abraldes JG, Duarte-Rojo A, Louvet $A$, et al. A histologic scoring system for prognosis of patients with alcoholic hepatitis. Gastroenterol. 2014;146(5):1231-9. doi:10.1053/ j.gastro.2014.01.018. e1-6.

41. Classification and Coding. Canadian Institue of Health Information. http://www.cihi.ca/ClHI-ext-portal/internet/EN/TabbedContent/standards+and+ data+submission/standards/classification+and+coding/cihi010689. Accessed May 2014.

\section{Submit your next manuscript to BioMed Central and take full advantage of:}

- Convenient online submission

- Thorough peer review

- No space constraints or color figure charges

- Immediate publication on acceptance

- Inclusion in PubMed, CAS, Scopus and Google Scholar

- Research which is freely available for redistribution 